\title{
Anodic Porous Alumina Array for Cyanine Fluorophore Cy3 Confinement
}

\author{
Larosa $\mathrm{C}^{* 1,2}$, Terencio $\mathrm{T}^{3,4}$, Converti $\mathrm{A}^{2}$ and Eggenhöffner $\mathrm{R}^{1}$ \\ ${ }^{1}$ Department of Surgical Science and Integrate Diagnostic University of Genoa, Italy and INBB \\ ${ }^{2}$ Department of Chemical and Process Engineering, University of Genoa, Genoa, Italy \\ ${ }^{3}$ Center for Micro-BioRobotics (MBR), Istituto Italiano di Tecnologia (IIT), Pontedera, Pisa, Italy \\ ${ }^{4}$ Department of Neuroscience and Brain Technologies (NBT)/Synaptic Neuroscience, Italy
}

${ }^{*}$ Corresponding author: Larosa C, Department of Surgical Sciences and Integrated Diagnostic, University of Genoa, largo Rosanna Benzi, E-mail: Claudio.Larosa@unige.it

Citation: Larosa C, Terencio T, Converti A, Eggenhöffner R (2014) Anodic Porous Alumina Array for Cyanine Fluorophore Cy3 Confinement. J Mater Sci Nanotechnol 1(1): S109. doi: 10.15744/2348-9812.1.S109

Received Date: June 03, 2014 Accepted Date: June 10, 2014 Published Date: September 08, 2014

\begin{abstract}
Self-organized anodic porous alumina films with hexagonal pore lattice have attracted a considerable attention for biological arrays and confinement of various organic probes dyes in solutions. A molecular structure with axial symmetry in bis-heterocyclic indole chains and conjugate system, such as cyanine fluorophore Cy3 dye, was investigated here with respect to its fluorescence when loaded in the anodic alumina pores. Cyanine $\mathrm{Cy} 3$, stabilized in a buffer phosphate at $\mathrm{pH} 7.3$, was dispersed in milli-Q water by assisted sonication to obtain a resulting concentration of $50 \mathrm{mM}$. The dark-pink solution was deposited slowly by casting method on the anodic porous alumina surface. Irradiation by mercury lamp at $530 \mathrm{~nm}$ wavelength was used to promote the electron transitions to upper discreet orbitals. The photon emission from this excited state was observed as fluorescence in real time from the samples. Atomic force microscopy was used to investigate the topography of anodic porous alumina before the use and it was compared with the CCD camera-microscope images. Frequency histograms showed significant reusable surface after four-cycle rinse steps in a selected surface area.
\end{abstract}

Keywords: Cy3; Anodic porous alumina; Dye; Fluorophore

\section{Introduction}

In the recent years, the engineering of anodic porous alumina (APA), produced by specific electrochemical routes, has found different applications in biophysical research. Masuda and Fukuda performed the first aluminium anodization almost twenty years ago with a long reaction time exceeding ten hours [1], a procedure that has not significantly changed so far. In particular, the electrochemical process leads to a surface with highly hexagonal pore order with unit cells of size in the range of 50-500 $\mathrm{nm}$ and above. The reduced dimension of APA pores has also attracted other several groups of researcher for the confinement of polymer, nanoparticles and materials [2-4]. A further and considerable attention was devoted to exploit multi-pore series as a potentially advanced array to contain biological materials [5-6] and, more generally, to the development of proteomics [7]. Further, attention was paid to the development of liquid phases matching the need to maintain the correct hydration of proteins in solution and in the cavity pores. In these conditions, proteins experience an APA amorphous environment with notable filling effects and emptying procedures evidenced mainly by fluorescence measurements.

In future approaches, proteins labelled by means of biological probes can be investigated through a gene protocol expression path exploiting enzymatic tests easily and accurately [8-10]. The successful use of APA pores to contain an organic cyanine solution of fluorophore Cy3 from Milli-Q water dispersion is verified in this work along with the opportunity to remove completely the Cy3 fluorescent probe and to reutilize potentially the matrix of APA pores.

\section{Experimental}

Aluminium foils $99.99 \%$ purity (Goodfellow) were degreased in ethanol with an analytical pure grade of $99 \%$ afterward, samples were kept at $400{ }^{\circ} \mathrm{C}$ for 4 hours in an oven for the thermal annealing. After this primary procedure of partial oxidation the aluminium was cooled down to room temperature and etched with a mixture of perchloric acid and ethanol in 1:4 ratio and at current density of $15 \mathrm{~A} / \mathrm{dm}^{2}$. This procedure was performed in a controlled temperature apparatus with a freezing mixture of ice and salts to control the temperature and to prevent metal burning.

During annealing there is a change of physical properties as strength and hardness of the microstructured material [11]. As well know, these physical changes make softer aluminum foils to undergo etching in the anodizing process. Various stress annealing and aluminum anodization affects the pore order with a tendency to give cracks and delamination. Such stress gives rise to volume expansion of the aluminium sheets. 
We used a solution of oxalic acid $0.5 \mathrm{M}$ for the anodization process divided in two-steps [12] preceded by a cleaning electrochemical polishing through a mixture solution of ethanol and perchloric acid 4:1. After this procedure the samples were rinsed accurately with Milli-Q water and sonicated for 15 minutes to remove surface impurities and residual debris in the pores. The samples were dried under $\mathrm{N}_{2}$ gas flow for 1 minute and stored in a dust-free box. The resulting template from the above procedures was used as anodic electrode during the preparation of the anodic porous alumina in homemade two-electrode electrochemical cell with oxalic acid $0.5 \mathrm{M}$ as electrolyte. Other conditions of the electrochemical treatment were bath temperature in a range $20 \div 5{ }^{\circ} \mathrm{C}$, electrode distance of $1.3 \mathrm{~cm}$, anodizing voltage of $50 \mathrm{~V}$ and current density of $0.5 \mathrm{~A} / \mathrm{dm}^{2}$ for 120 minutes. In our APA preparations the cathode was a platinum sheet.

In view of the insulator behaviour of alumina, morphological and topographical parameters were obtained through AFM investigations, using Innova Veeco instrument model with NSC18 (MikroMasch, Italy) probes in non-invasive tapping mode. A Silicon conical tip with a typical radius of curvature of $10 \mathrm{~nm}$ and an aspect ratio about 3:1 was used. The samples were imaged under tapping observation mode with an average spring constant of the probe cantilever of $17 \mathrm{~N} / \mathrm{m}$. In tapping mode the cantilever vibrates at a driving frequency slightly lower than the peak value of its resonant frequency, between $60-90 \mathrm{kHz}$.

The sample was characterized on a glass slide surface anchored with two epoxy resin spots. We used a square-shaped sample of APA, with dimension of $0.5 \times 0.5 \mathrm{~cm}^{2}$. With a Gilson micropipette, we have cast deposited $10 \mu \mathrm{l}$ drop by drop of a solution $50 \mathrm{mM}$ of Cy3 at room temperature at time 0 and each rinse with10 $\mu$ l Milli-Q water a new CCD-camera frame image was acquired. Cy3 was supplied from Sigma-Aldrich, Inc.

The frame images are acquired with a CCD camera on the optical microscope in a dust-free zone at night; the CCD cameramicroscope is supported on a pneumatic antistatic support, to avoid interference from vibration effects during the running phase of data acquisition. We have used a 100x magnification, 0.95 numerical aperture air objective, with 0.32 mm working distance. The mercury vapour lamp in use has various spectral emission lines with a peak at $530 \mathrm{~nm}$; it was switched on at least one hour before the use. The CCD camera has $1600 \times 1200$ pixels, each $7.4 \mu \mathrm{m}^{2}$ size. Since the lamp has a power of $60 \mathrm{~W}$ we can estimate the irradiance of $\sim 1300 \mu \mathrm{W} / \mathrm{cm}^{2}$ on the APA surface at a distance of $70 \mathrm{~cm}$. The sensor exposure time is controlled by diaphragm aperture fixed at $187 \mathrm{~ms}$ suitable to a Cy3 concentration of $50 \mathrm{mM}$; deposited by cast way on APA surface area of $4 \times 4 \mathrm{~mm}^{2}$. The Cy3 employed in the present work is brighter and more photo stable than many other fluorophores and it has the capability to emit photons with longer wavelength around $590 \mathrm{~nm}$ in a period of $10^{-9}-10^{-8} \mathrm{~s}$ after green light excitation of $530 \mathrm{~nm}$. After different attempts, we have used for our experiments a molar concentration in the range $10^{-5} \div 10^{-4} \mathrm{M}$ to avoid saturation effect and with a molar extinction of $1.5 \times 10^{5} \mathrm{Mcm}^{-1}$. The latter value is acquired by dispersion of the organic dye in methanol solvent.

\section{Results and Discussion}

\section{Chemistry of APA formation}

Aluminium is an electropositive element with a natural tendency to give passivation. Its reactivity to oxygen induces the formation of aluminium oxide as described from reaction (1) and with water to give metal oxide and hydrogen evolution (2) in both annealing and electrolytic process:

$$
\begin{array}{ll}
4 \mathrm{Al}(\mathrm{s})+3 \mathrm{O}_{2}=2 \mathrm{Al}_{2} \mathrm{O}_{3}(\mathrm{~s}) & \Delta \mathrm{G}^{\circ}=-1582 \mathrm{~kJ} / \mathrm{mol} \\
2 \mathrm{Al}(\mathrm{s})+9 \mathrm{H}_{2} \mathrm{O}(\mathrm{l})=\mathrm{Al}_{2} \mathrm{O}_{3}(\mathrm{~s})+6 \mathrm{H}_{3} \mathrm{O}^{+}+6 \mathrm{e}^{-} & \Delta \mathrm{G}^{\circ}=-871 \mathrm{~kJ} / \mathrm{mol}
\end{array}
$$

In the annealing, the oxygen partial pressure in the oven induces a preliminary metal oxidation. The etching reduces the roughness of the surface; large ridges, small irregular aggregates with micron and submicron dimensions are removed; in our working conditions the ratio between reagents in the mixture for etching does not subtract completely the aluminium oxide. The electrode template designed from first layer oxide and an aluminium plate was used as starting material for the electrolytic anodization. Electrical anodization increases the depth inside the hexagonal honeycombs with eventual regular pores inside their deep walls.

We focused on the effects of electrochemical parameters on the surface morphology. Anodization voltage was varied in the range $25 \div 55 \mathrm{~V}$ in DC; accordingly, the pore average size grew from $700 \mathrm{~nm}$ up to $5 \mu \mathrm{m}$. In addition, the selected electrochemical environment affects the pore dimensions: oxalic acid leads to larger pore with respect to, for instance, sulphuric acid. The electrode distance in the range $1 \div 3 \mathrm{~cm}$ is relevant to define the pore density; when the distance is $1.5 \mathrm{~cm}$ the pore-pore average distances is $1.5 \mu \mathrm{m}$. The pore depth depends on electrolytic duration process. The pore depth changes from $200 \mathrm{~nm}$ up to $700 \mathrm{~nm}$ when the anodization time changes from 30 to 120 minutes.

\section{Physical chemistry of Cy3 Fluorophore}

In Figure 1 we report the structure of the Cy3 hydrocarbon Indole molecule. In Table 1 we report the parameters that describe the conditions involved in the excitation and emission of such fluorophore. The presence of a sulfonate group in the aromatic rings in position 5 and 5 ' is used to improve the water solubility and to reduce the dye aggregation. In particular, the introduction of su- 
lfonate groups induces a negative charge on the dye molecule with the tendency to support a hydrophilic state. Furthermore, the interaction between molecules strongly hinders the fluorescence yields as the result of dimers-trimers formation, a process limited by sonication applied to the solution. The concentration of Cy3 dye in a solution with the camera diaphragm aperture of $187 \mathrm{~ms}$ and with its fluorescence yield on the APA sample are sources of variability correlated by inverse proportionality dependence. The fluorescence emission of APA and Cy3 dye requires the use of a narrow band-pass emission filter to acquire the signal. In particular, the adopted Cy3-HYQ filter reduces the effect of light scattering and permits only a narrow bandpass between $530 \div 560$ $\mathrm{nm}$ on the total incoming light. Namely, such Cy3-HYQ Nikon filter is used in experimental conditions of double fluorophore signals due to difficulty to discriminate for instance two fluorescence contributions from Cy3-Cy5 or a single fluorophore signal with a second contribution from the matrix itself. Adjusting the band pass solved this problem.

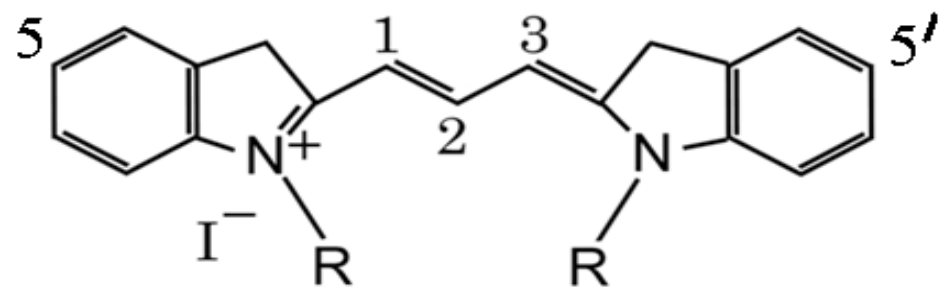

\section{Cy3}

Figure 1: Symmetric hydrocarbon chemical structure of the Cy3 dye, Indole with unsaturated conjugates double bonds. Two aromatic positions in 5 and 5 ' are occupied by sulfonate substituent.

\begin{tabular}{|c|c|c|c|c|c|c|}
\hline $\begin{array}{c}\text { Probe } \\
\text { Name }\end{array}$ & $\begin{array}{c}\text { Excitation } \\
\text { wavelength } \\
(\mathbf{n m})\end{array}$ & $\begin{array}{c}\text { Emission } \\
\text { wavelength } \\
(\mathbf{n m})\end{array}$ & $\begin{array}{c}\text { Molecular } \\
\text { weight } \\
(\mathbf{D a})\end{array}$ & $\begin{array}{c}\text { Quantum } \\
\text { yield }\end{array}$ & $\begin{array}{r}\text { Molar } \\
\text { extinction } \\
\text { Coefficient } \\
\left(\mathbf{M} \times \mathbf{c m}^{-1}\right)\end{array}$ & $\begin{array}{r}\text { Cy3 Lifetime } \\
\boldsymbol{\tau}(\mathbf{s})\end{array}$ \\
\hline Cy3 & $512-550$ & $570-615$ & 767 & 0.15 & Dark-pink & 150.000 \\
\hline
\end{tabular}

Table 1: Organic fluorescent cyanine Cy3 dye and related properties

\section{AFM imaging of APA surface}

The electrolytic process, described in section 'Chemistry of APA formation', is able to obtain hexagonal structures with a mean diameter of 1-3 $\mu \mathrm{m}$ as reported in Figure 2. The pore depth with a size of $700 \mathrm{~nm}$ approximately allows the filling of the structure; the pore dimensions and the hole inter-distance are shown in the same Figure 2. The combined effect of annealing and electrochemical treatments gives hexagonal pores significantly closer and larger pores than the common values reported in the current scientific literature [13] as required for the suggested application.

Figure 2 a shows the hexagonal ordering around a central pore, although irregular with protruding tips (white in the original scale) and buried holes (almost red-brown in the same scale). Walls morphological details are reported in both Figure 2a and Figure $2 \mathrm{~b}$, where regular indented cleavage fingerprints are shown in the area around the sigle pore and in the wide area reported, respectively. The intrinsic corrugation of the hexagonal edges is clearly shown by the profile in Figure $4 \mathrm{c}$ where the profile on the perimetral line including the six sides is reported.

A clear shape of a pore hexagonal structure and of their surface arrangement is given in Figure 2a where a plot line across the surface is shown with a definition of different conical shape instead of the expected cylindrical shape. This deviation reflects the inability of the tip to penetrate inside the pores prevented by the tip geometry and curvature [14]. Further, it appears clearly that the structure of hexagonal edges at the surface is not perfectly flat and deviates from the expected cylindrical shape from base to edge [15] towards a conical profile. The profiles on the lines in Figure 2 are reported in Figure 2d,e,f; in Figure 2d along a straight line crossing a pore wall at its centre (white line in Figure 2a), in Figure 2e in the wide area in Figure 2b, in Figure 2a the perimetral profile along the six sides of the hexagonal pore (green line in Figure 2a).

In case that the APA formation effect is purely electrochemical, the result is a direct proportionality between applied voltage, average pore size (diameter), and average interpore distance. In our case the latter parameter is due to a different mechanism, i.e. the annealing procedure. Clearly, the order of pore arrangement occurring in the electrochemical-only treatment is lacking in the thermal annealed samples. It has also been hypothesized that the Al erosion rather than oxide formation explains the surface patterning with so large pores. However, we checked that the resulting surface was insulating and, additionally, we also performed an electrolytic test experiment in which annealed Al metal sheets were employed as both cathode and anode. At the end of the latter process, a different surface morphology appeared for cathodic Al with respect to anodic Al. Instead of closely packed pores, the cathodic $\mathrm{Al}$ showed only prominences approximately $2 \mu \mathrm{m}$ apart with dimensions of $1.0 \pm 0.5 \mu \mathrm{m}$ (supplementary data available on request). 

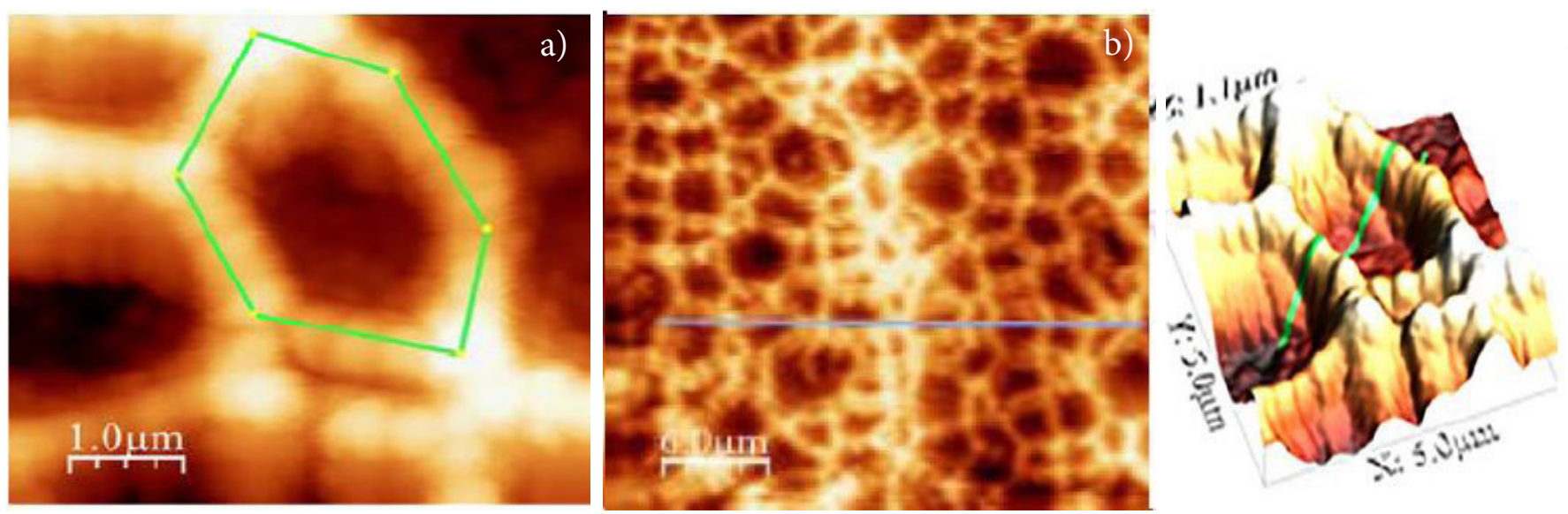

c)

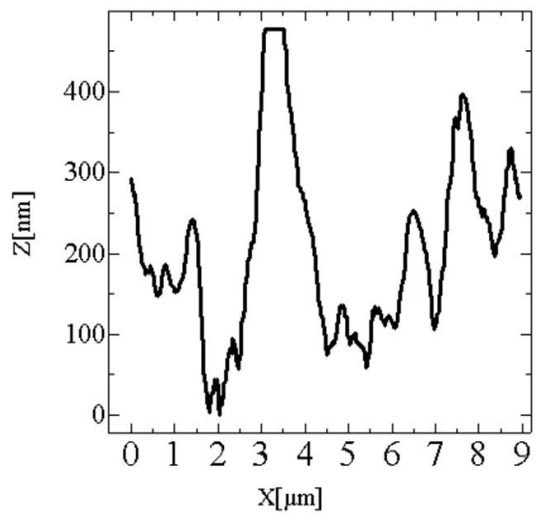

d)

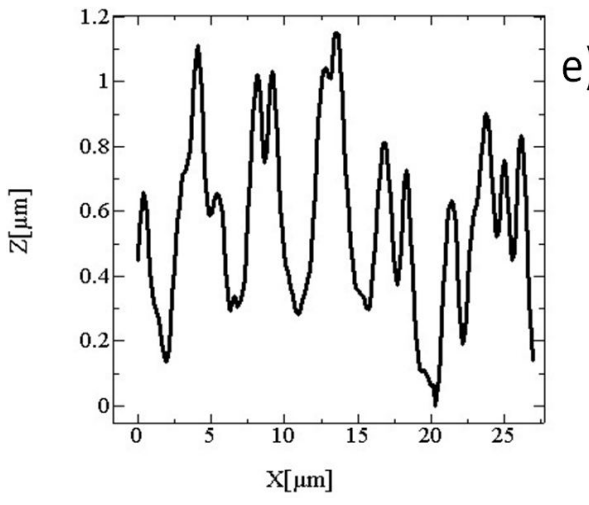

e)

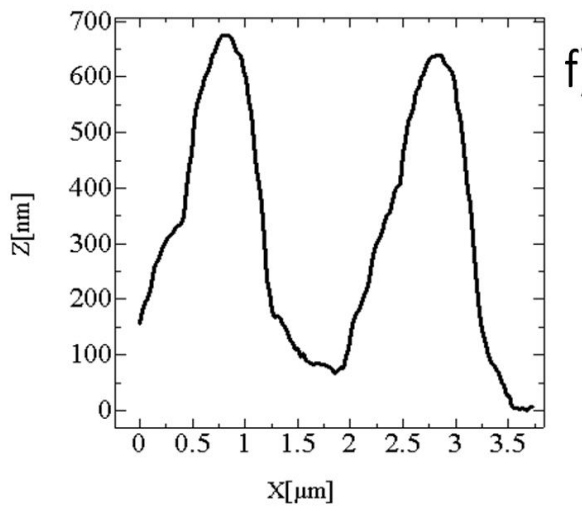

Figure 2: a) 2D AFM image of porous alumina surface after electrolytic synthesis process, reported at the scanning area of $5 \times 5 \mu \mathrm{m}^{2}$; and b) $30 \times 30 \mu \mathrm{m}^{2}$ scanning area. The lines indicate the directions selected for the profile analyses. c) 3D AFM image corresponding to the same area explored in Figure 2a. in Figure 2d-f AFM profiles of the anodic porous alumina are reported along the directions shown in Figure 2a-c.

\section{CCD fluorescence microscopy}

The top surface of Cy3 loaded APA was characterized by CCD camera-microscope with dedicated acquisition software Axio Visio 3.1. ImageJ free software was used to extract the photo luminescent intensity from frame images with 8-bit data and size of 338 $\times 320$ pixels and to express it in frequency histograms. The histograms report on the $\mathrm{x}$ - axis an arbitrary grey scale colour; in the $y$-axis the frequency photon emission events. These two numbers are the minimum and maximum displayed pixel values and define the display range. ImageJ displays images by linearly mapping pixel values in the range $0 \div 255$. Pixels with a value less than the minimum are displayed as black and those with a value greater than the maximum are displayed as white whereas the number of event that presents such intensity is reported on the y-axis. We can split the experimental procedure for data acquisition from the CCD microscope in three steps: 1) acquire few images as reference profile with empty APA; 2) acquire images immediately after the irradiation of APA/Cy3 fluorophore composite to mercury lamp and finally 3) complete three runs of cycles of drying and making APA empty again. These working steps are summarized in the schematic of the experimental procedure reported in Figure 3.

The fluorescence emission, induced by a vapour mercury lamp, from the Cy3 dye deposited inside pores stimulate a transition of electrons on a secondary upper orbital with higher energy. In a few nano-seconds electrons return to ground level in one or more pathways. The last decay emits light at the wavelength of $560 \mathrm{~nm}$ at the centre of the bandpass filter. This light is acquired in realtime by the CCD camera.

We can distribute the global contribution of fluorescence into three effects: organic dye, traces of crystalline oxalic acid commonly residual within the pores after the normal electrochemical process [16]; oxygen vacancies formed during the preparation of APA. The second contribution is minimized by our accurate rinse of the matrix by water immersion and $\mathrm{N}_{2}$ gas drying and it is absent in the final fluorescence.

We report three selected different frame images in Figure 4 from our experimental procedure discussed above. In particular, we report from the above mentioned frame images two series of histograms. The first histograms are acquired from the whole area image; the second ones on the same images but in a much smaller area corresponding approximately to a single pore region. These histogram levels are representative of the emission from around and inside a micro pore before mercury lamp irradiation and with- 
out fluorophore in Figure 4a-c; of the confinement of Cy3 fluorophore in single pores in Figure 4d-f; of the degree of fluorophore removal Figure $4 \mathrm{~g}$-i. The histograms are studied to evaluate the confinement and emptying procedures of organic liquid dye in the phases as reported in Figure 3.

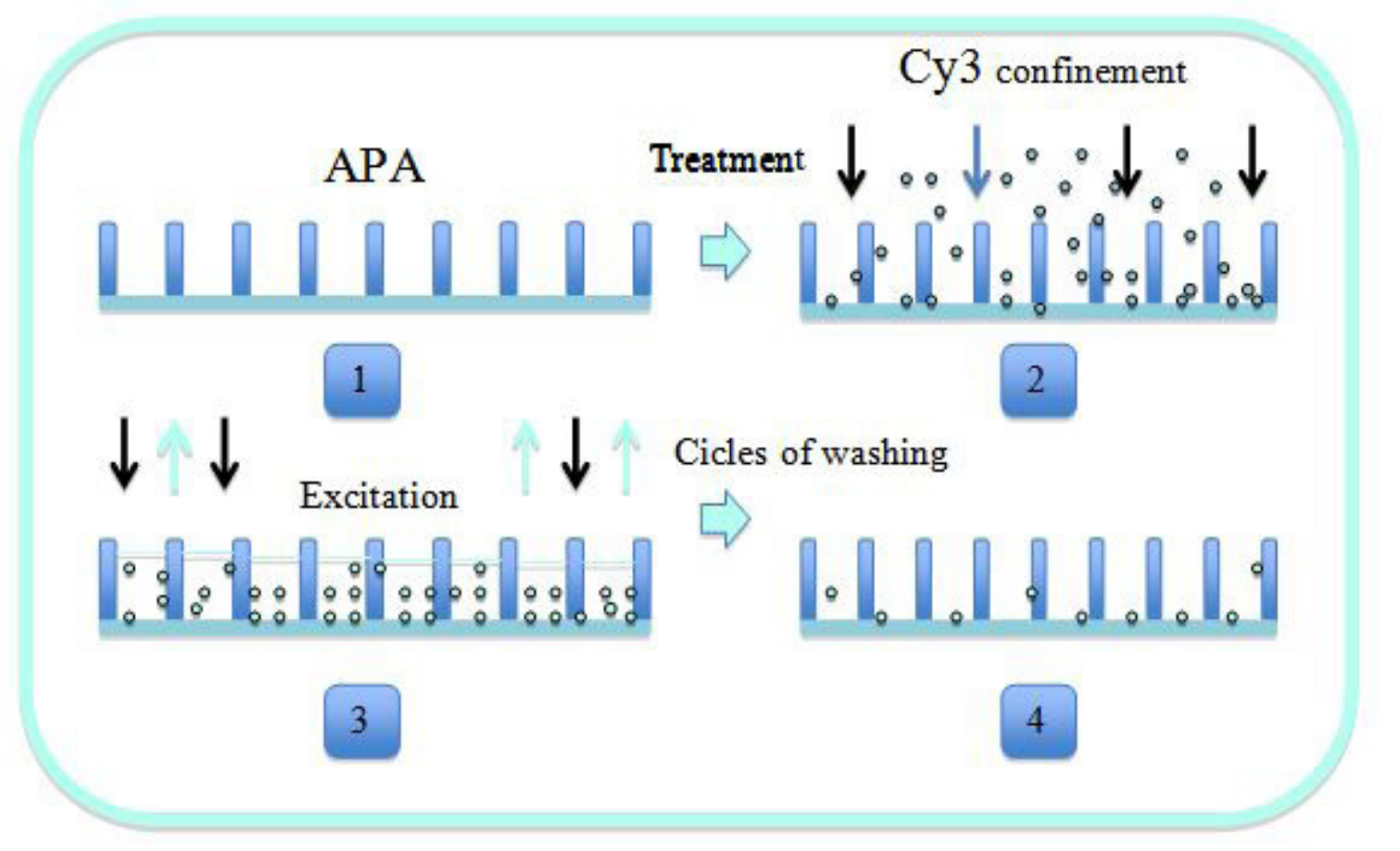

Figure 3: Draft scheme of the confinement procedure of Cy3 molecules on and inside anodic porous alumina.

The pore dimensions as obtained from the reference image acquired by the CCD-microscope reported in Figure 4 are in close agreement with the scale values resulting from AFM images in Figure 2. The pore number on the APA surface is estimated of the order of $1 \div 2 \times 10^{7} \mathrm{~cm}^{-2}$ with sides of the hexagonal structure around $1 \mu \mathrm{m}$ and an inter-distance diameter of $3 \mu \mathrm{m}$. Such $3 \mathrm{D}$ nanostructured surface with high number of pores per unit area offers the possibility to screen a huge number of biological samples in each spot, for instance during the gene expression of free proteins in the protocol of the nucleic acid programmable protein array (NAPPA) [17]. The peak of the histogram occurs around the mean value of 44 over 256 positions on the x-axis with a standard deviation of approximately 5 , as shown in Figure $4 \mathrm{~b}$. The mean values observed both around a single pore and over wide areas are fairly close to the beginning of the x-scale, indicating low fluorescence levels. The analysis of a much smaller region corresponding to single pores gives results without significant deviations from the wide area analyses, as shown in Figure 4c, with a tendency, however, to give slightly higher values from the short range. The APA surface in the experimental conditions of Figure 4, as discussed above, is free of $\mathrm{Cy} 3$, suggesting a residual luminescence of native APA surface with respect to ambient white light with small yet non-zero contributions also at wavelengths compatible with the Cy3 signals and admitted thus to pass the optical filter.

We can propose that the nano-pore axial symmetry and ordering led to the Cy3 dye orientation predominantly along the pore axis as a result of the capillary force from the APA surface to the pore wall governing the physical filling. Such process would resemble the Langmuir-Blodgett technique inducing one or more monolayer of an organic material in a specific ordered state. The average size of the Cy3 dye molecule is approximately 1-2 nm, i.e. two orders of magnitude smaller than the pore average diameter of the APA surface. The volume expansion from Al metal to alumina would cause high stress [18], which leaves a lot of voids with a size of about 1-2 nm buried within APA matrix and cracks with size of $5 \mathrm{~nm}$ extending from the surface to internal voids [19-20]. During the drop-cast, molecular dye diffuses into the nano-voids through cracks. Each nano-void can contain one or two dye molecules by reducing the dipole-dipole interaction between $\mathrm{Cy} 3$ group. Also the high dielectric constant of alumina reduced the interaction between Cy3 in neighbouring nano-voids [21]. The dipole-dipole interaction between polar groups would induce the formation of un-emissive dimers, trimers and Cy3 organic dye aggregates during the sample preparation. Assisted sonication was adopted to reduce such unwanted effects on the final preparation solution, since agglomeration reduces the quantum yield fluorescence. As shown in Figure 4d-f, the luminescence signal collected in the conditions of full cast deposition of the Cy3 dye on the APA surface and after mercury lamp exposure, gives the main peak approximately at the middle of the light grey scale. This result can be partly ascribed to the above effects, in addition to the low concentrations employed to avoid saturations. As shown in Figure $4 \mathrm{e} v \mathrm{f}$, the small areas are darker than the whole area. 


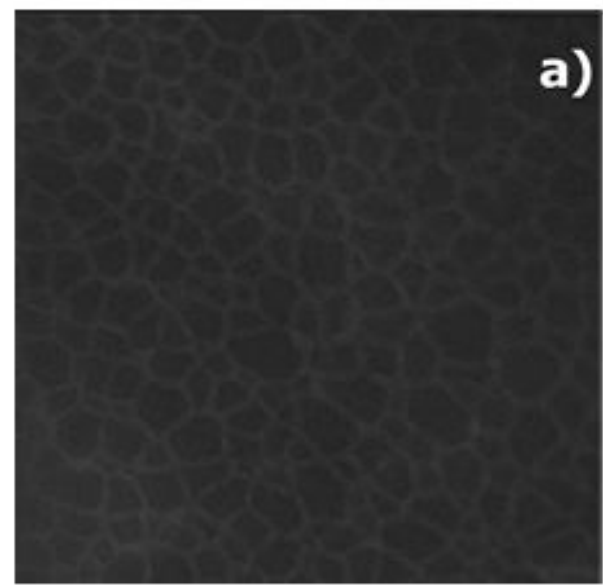

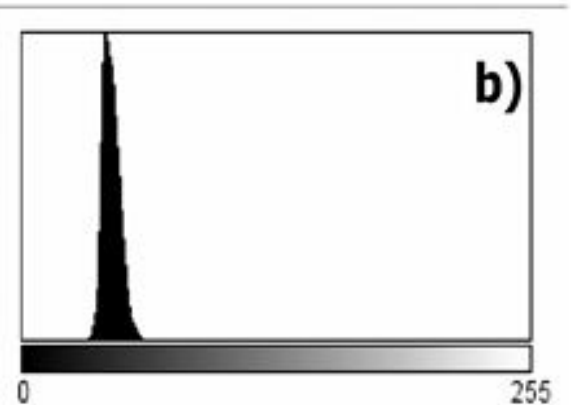

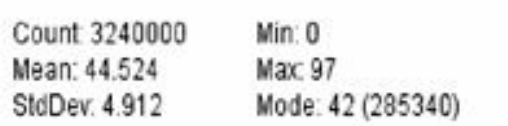

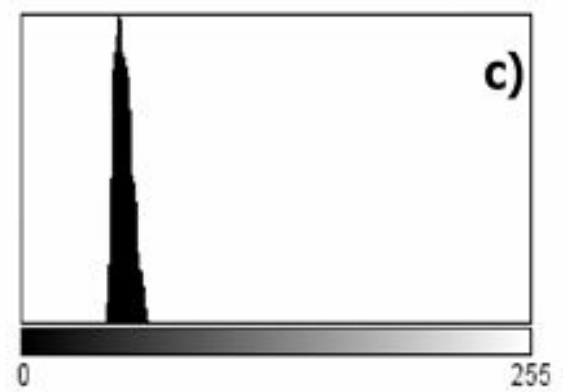

Count 36018

Mean: 50.414

StdDev. 4.343
$\operatorname{Min}: 41$

Max: 63
Mode: $48(3131)$

Figure 4 a,b,c. a) CCD camera image at 100x of APA acquired as reference; the width of the image is $65 \mu \mathrm{m}$; b) histogram on the whole image in Figure $4 \mathrm{a} ; \mathrm{c}$ ) histogram from a selected small region in Figure 4a corresponding approximately to a single pore, i.e. $3 \mu \mathrm{m}$ in linear size.

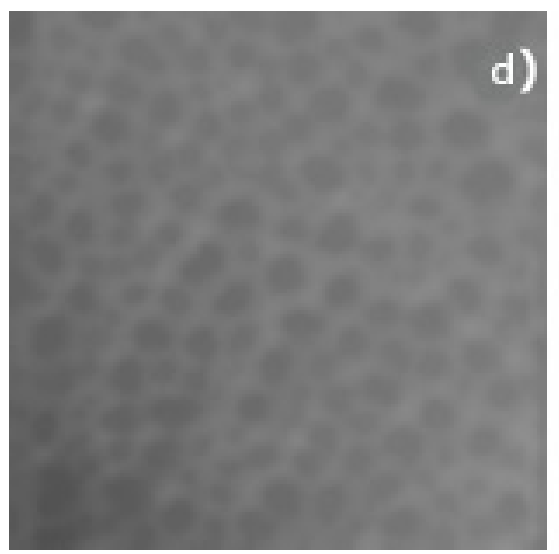

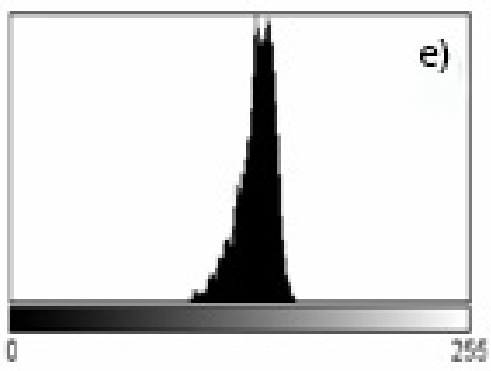

Count $3240000 \quad$ Virc 97

Mean:136.761 Nax162

Stcoer $10.241 \quad$ Nose.137 (147369)

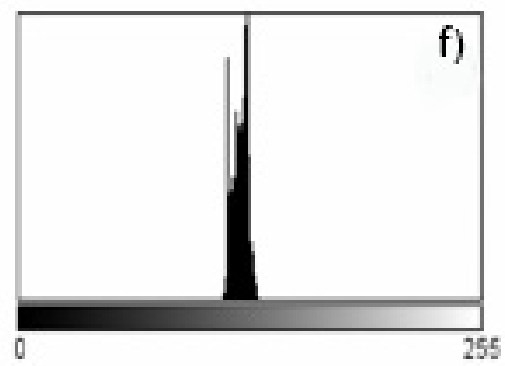

Cosnt $\mathbf{4 9 9 5 0}$

Nean: 121.914

Mn: 110

Max 134

Mode: 126 (5698]

Figure 4 d,e,f. d) CCD camera image at 100x of the APA surface with Cy3 cromophore; the width is $65 \mu \mathrm{m}$; e) histogram of Cy3 cromophore fluorescence after exposition to mercury lamp on the whole image in Figure $4 \mathrm{~d}$; histogram of fluorescence from a small region in Figure $4 \mathrm{~d}$ corresponding approximately to a single pore, i.e. $3 \mu \mathrm{m}$.
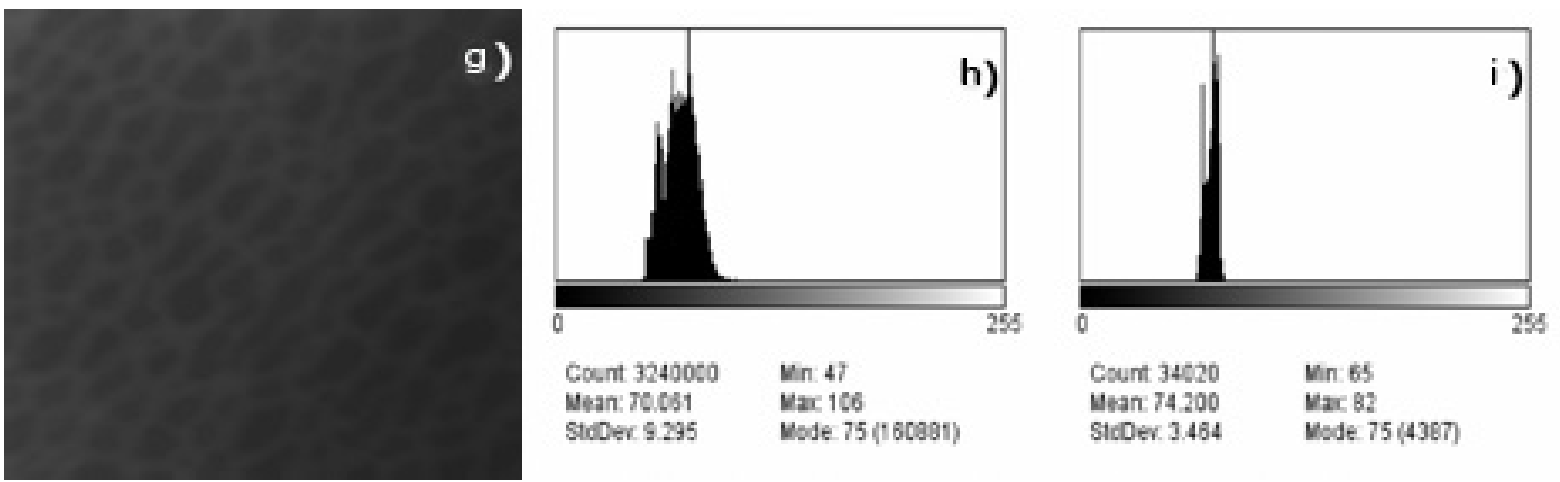

Figure 4: g,h,i. g) CCD camera image at 100x of the APA surface after Cy3 confinement and four rinse cycle treatment to remove the fluorophore; h) histogram of residual fluorescence on the whole image in Figure 4g; i) histogram of fluorescence from the small region in Figure 4g corresponding approximately to a single pore.

The effects shown in Figure 4g-i were collected after four rinse cycles with the aim to remove the dye molecules. As shown in Figure $4 \mathrm{~g}$ the global fluorescence moves towards lower values as required, i.e. close to the native state without further treatments. We have examined also the emission of light from areas well inside the pores and at the edges of the hexagonally distributed peaks; the results of the histogram data averages are reported in Table 2. The fluorescence reduction rate is higher at the edges with respect to the middle of the pores. These averages are stable and only marginally affected by positions over the whole area and by the width of the area in selected regions inside and at the edges of the peaks. Such results are quite promising for applications in which the fluorophore is attached to specific proteins or other biological substances confined in the pores by chemical bonds, whereas the unbound fluorescent molecules need to be washed out, in particular if positioned at the top of the edges, which are seen with high optical efficiency by CCD sensors. 


\begin{tabular}{|c|c|c|c|}
\hline & $\begin{array}{c}\text { Average on } 65 \mu \mathrm{m} \mathrm{x} \\
65 \mu \mathrm{m} \text { area }\end{array}$ & Edge of the pores & Inside the pores \\
\hline Native APA & $44 \pm 5$ & $48 \pm 4$ & $39 \pm 3$ \\
\hline APA with Cy3 & $137 \pm 10$ & $146 \pm 3$ & $127 \pm 3$ \\
\hline $\begin{array}{c}\text { After four } \\
\text { rinses }\end{array}$ & $70 \pm 9$ & $75 \pm 4$ & $70 \pm 5$ \\
\hline
\end{tabular}

Table 2: Peak positions in the range $0 \div 255$ (from black to white).

\section{Conclusion}

We reported about APA fabrication which, thanks to a special annealing procedure, produced extraordinary large pore size, in the range of 1-2 $\mu \mathrm{m}$. Our results from AFM and CCD-camera characterizations show the presence on APA surface of micrometric pores useful for possible confinement and eventual rinse of Cy3 organic dye on the same APA surface. The fluorescence emission on APA performed in the present work opens new intriguing aspects about the layer or multilayer confinement of Cy3 organic liquids dye and the filled pores. The capillary force during the cast deposition plays a role during the pore filling with nano-metric size dimension of the pore defects. These effects have an influence also on the confinement of the dye in the nano-void included in the walls of the hexagonal pore cells with dimers and trimers effects of desegregation. The details of the fluorescence confinement of $\mathrm{Cy} 3$ in this intrinsic cavity and the definition of the quantum yield are possible topics for further investigations.

\section{Acknowledgement}

The authors are grateful to COST actions MP1105 and MP1206 on flame retard and electro-spinning, respectively. CL acknowledges also COST actions for the support in Short Term Scientific Mission.

\section{References}

1. Masuda H, Fukuda K (1995) Ordered metal nanohole arrays made by a two-step replication of honeycomb structures of anodic alumina. Science 268: 1466-8.

2. Gultepe E, Nagesha D, Menon L (2007) High-throughput assembly of nanoelements in nanoporous alumina templates. Applied Physics Letters $90: 163119$.

3. Toccafondi C, Stępniowski WJ, Leoncini M, Salerno M (2014) Advanced morphological analysis of patterns of thin anodic porous alumina. Materials Characterization 94: 26-36.

4. Thorat S, Diaspro A, Scarpellini A, Povia M, Salerno M (2013) Comparative Study of Loading of AnodicPorous Alumina with Silver Nanoparticles Using Different Methods. Materials 6: 206-16.

5. Grasso V, Lambertini V, Ghisellini P, Valerio F, Stura E, et al. (2006) Nanostructuring of a porous alumina matrix for a biomolecular microarray. Nanotechnology 17: 795-8.

6. Matsumoto F, Kazuyuki N, Masuda H (2004) Flow-through-type DNA array based on ideally ordered Anodic porous alumina substrate. Adv. Mater 16: 2105-08.

7. Ramachandran N, Raphael J V, Hainshwort E, Demirkan G, Fuentes MG, et al. (2008) Next-generation high-density self-assembling functional protein arrays. Nature Methods 5: 535-8.

8. Nicolini C, Correia TB, Stura E, Larosa C, Spera R, et al. (2013) Atomic Force Microscopy and Anodic Porous Alumina of Nucleic Acid Programmable Protein Arrays. Recent Patents on Biotechnology 7: 112-21.

9. Salerno M, Giacomelli L, Larosa C (2011) Biomaterials for the programming of cell growth in oral tissues: The possible role of APA. Bioinformation 5: 291-3. 10. Lee JR, Magee DM, Gaster RS, LaBaer J, Wang SX (2013) Emerging protein array technologies for proteomics. Expert Rev Proteomics 10: 65-75.

11. Xu WL, Zheng MJ, Wu S, Shen WZ (2004) Effect of high-temperature annealing on structural and optical properties of highly ordered porous alumina membrane. Appl Phys Lett 85: 4364.

12. Thompson GE (1997) Porous anodic alumina: fabrication, characterization and applications. Thin Solid Films 297: 192-201.

13. Ono S, Saito M, Asoh H (2005) Self-ordering of anodic porous alumina formed in organic acid electrolytes.Electrochemical Acta 51: 827-33.

14. Sui Y, Saniger JM (2001) Characterization of anodic porous alumina by AFM. Material Letters 48: 127-36.

15. Diggle JW, Downie TC, Goulding CW (1969) Anodic oxide films on aluminium. Chem Rev 69: 365-405.

16. Gao T, Meng G, Zhang L (2003) Blue luminescence in porous anodic alumina films: the role of the oxalic impurities. J Phys C 15: 2071-9.

17. Sartore M, Eggenhoffner R, Bezerra T, Stura E, Hainsworth E, et al. (2010) Label-free detection of NAPPA via atomic force microscopy. In: Functional Proteomics and Nanotechnology-based Microarrays, London: Pan Stanford Series on Nanobiotechnology 2: 109-20.

18. Jessensky O, Muller F, Goesele U (1998) Self-organized formation of hexagonal pore arrays in anodic alumina. Appl Phys Lett 72: 1173.

19. Macdonald DD (1993) On the formation of voids in anodic oxide films on aluminium. J Electrochem 140: L27-30.

20. Mei YF, Wu XL, Shao XF, Huang GS, Siu GG (2003) Formation mechanism of alumina nanotube array. Phys Lett A 309: 109-13.

21. De Wit HJ, Wijenberg C, Crevecoeur C (1976) The dielectric breakdown of anodic aluminium oxide. Electrochem Soc 123: 1479-86. 


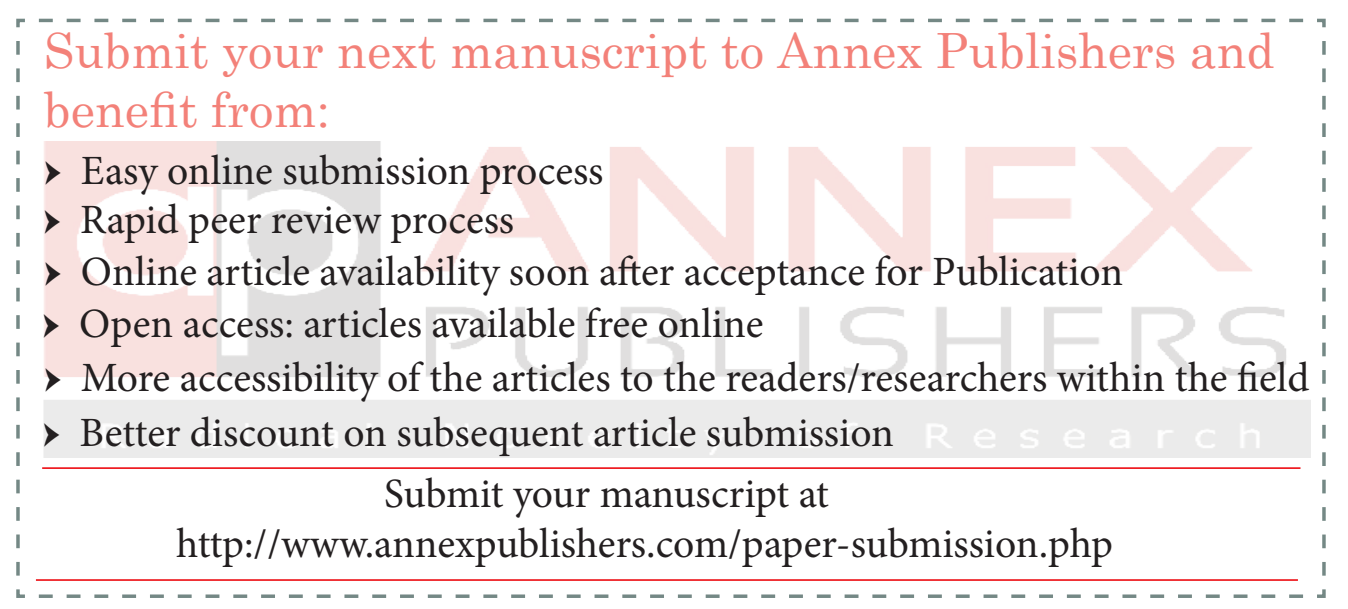

